\title{
Preoperative 2D-echocardiographic assessment of pulmonary arterial pressure in subgroups of liver transplantation recipients
}

\author{
Jungchan Park', Myung Soo Park², Ji-Hye Kwon' ${ }^{1}$, Ah Ran Oh', \\ Seung-Hwa Lee ${ }^{3}$, Gyu-Seong Choi ${ }^{4}$, Jong Man Kim ${ }^{4}$. \\ Keoungah Kim ${ }^{5}$, and Gaab Soo Kim ${ }^{1}$
}

'Department of Anesthesiology and Pain Medicine, Samsung Medical Center, Sungkyunkwan University School of Medicine, Seoul, ${ }^{2}$ Department of Medicine, Dongtan Sacred Heart Hospital, Hallym University School of Medicine, Hwaseong, ${ }^{3}$ Department of Medicine, Heart, Stroke, and Vascular Institute, Samsung Medical Center, Sungkyunkwan University School of Medicine, Seoul, 'Department of Surgery, Samsung Medical Center, Sungkyunkwan University School of Medicine, Seoul, ${ }^{5}$ Department of Anesthesiology, School of Dentistry, Dankook University, Cheonan, Korea

Received March 30, 2021

Revised July 5, 2021

Accepted July 21, 2021

\section{Corresponding author}

Gaab Soo Kim, M.D.

Department of Anesthesiology and Pain Medicine, Samsung Medical Center, Sungkyunkwan University School of Medicine, 81 Irwon-ro, Gangnam-gu, Seoul 06351, Korea Tel: 82-2-3410-0360

Fax: 82-2-3410-0361

E-mail: gskim@skku.edu

Jungchan Park and Myung Soo Park contributed equally to this study.
Background: The clinical efficacy of preoperative 2D-echocardiographic assessment of pulmonary arterial pressure (PAP) has not been evaluated fully in liver transplantation (LT) recipients.

Methods: From October 2010 to February 2017, a total of 344 LT recipients who underwent preoperative 2D-echocardiography and intraoperative right heart catheterization (RHC) was enrolled and stratified according to etiology, disease progression, and clinical setting. The correlation of right ventricular systolic pressure (RVSP) on preoperative 2D-echocardiography with mean and systolic PAP on intraoperative RHC was evaluated, and the predictive value of RVSP > $50 \mathrm{mmHg}$ to identify mean PAP > $35 \mathrm{mmHg}$ was estimated.

Results: In the overall population, significant but weak correlations were observed $(\mathrm{R}=$ 0.27 ; $P<0.001$ for systolic PAP, $R=0.24$; $P<0.001$ for mean PAP). The positive and negative predictive values of RVSP > $50 \mathrm{mmHg}$ identifying mean PAP > $35 \mathrm{mmHg}$ were $37.5 \%$ and $49.9 \%$, respectively. In the subgroup analyses, correlations were not significant in recipients of deceased donor type $L T(R=0.129 ; P=0.224$ for systolic PAP, $R=0.163 ; P=$ 0.126 for mean $P A P)$ or in recipients with poorly controlled ascites $(R=0.215 ; P=0.072$ for systolic PAP, $\mathrm{R}=0.21 ; \mathrm{P}=0.079$ for mean PAP).

Conclusions: In LT recipients, the correlation between RVSP on preoperative 2D-echocardiography and PAP on intraoperative RHC was weak; thus, preoperative 2D-echocardiography might not be the optimal tool for predicting intraoperative PAP. In LT candidates at risk of pulmonary hypertension, RHC should be considered.

Keywords: Catheterization; Echocardiography; Hypertension; Liver transplantation; Pulmonary; Swan-ganz.

This is an Open Access article distributed under the terms of the Creative Commons Attribution Non-Commercial License (http://creativecommons.org/licenses/by-nc/4.0) which permits unrestricted non-commercial use, distribution, and reproduction in any medium, provided the original work is properly cited.

Copyright (C) the Korean Society of Anesthesiologists, 2021 


\section{INTRODUCTION}

Pulmonary hypertension (PH) is not uncommon in endstage liver disease, and the presence of $\mathrm{PH}$ is of particular concern in liver transplantation (LT) $[1,2]$. The current diagnostic criteria for porto-pulmonary hypertension require hemodynamic measurements obtained via right heart catheterization (RHC): mean PAP (mPAP) > 25 mmHg, pulmonary vascular resistance $>3$ Woods units, and pulmonary capillary wedge pressure $<15 \mathrm{mmHg}$ [3]. Reportedly, the mortality of LT exponentially increased at the threshold of mean pulmonary artery pressure (PAP) over $35 \mathrm{mmHg}$ [4]. Thus, RHC, the gold standard for evaluating pulmonary hemodynamics in high-risk candidates, has been justified despite the invasiveness of the procedure associated with fatal complications such as bleeding and arrhythmia [5].

To select patients at risk, the current guidelines recommend echocardiographic assessment for screening for all LT candidates [6]. 2D-Echocardiography is a non-invasive, widely available, and relatively inexpensive diagnostic method [7], and previous studies have demonstrated its clinical efficacy in LT candidates $[8,9]$. Because $\mathrm{PH}$ that responds well to preoperative treatment is indicated for LT [1], continuous monitoring of PAP is crucial in liver allocation as well as screening of $\mathrm{PH}[10,11]$. 2D-Echocardiographic assessment has shown benefits in monitoring progression or improvement of $\mathrm{PH}$ [7], but reliability of preoperative 2D-echocardiography in predicting actual intraoperative PAP remains uncertain.

Our institution is a large-volume center with experience of more than 2,000 LT cases over 20 years. Preoperative $2 \mathrm{D}$-echocardiography is included in the routine preoperative evaluation and is performed and interpreted by echocardiographers and cardiologists. All intraoperative parameters measured by direct cannulation are recorded in the institutional LT database by attending anesthesiologists. This study evaluated the correlation between right ventricular systolic pressure (RVSP) on preoperative 2D-echocardiography and PAP measured by intraoperative RHC and whether preoperatively high RVSP could predict intraoperative MPAP $>35 \mathrm{mmHg}$.

\section{MATERIALS AND METHODS}

\section{Study population and data collection}

The Institutional Review Board at our institution approved this study and waived the need for individual consent (no. 2018-12-095-001). The study was conducted according to the principles of the Declaration of Helsinki. Study data were derived from the institutional LT database and retrospectively analyzed. From October 2010 to February 2017, 415 adult LT recipients with intraoperative RHC were enrolled in the registry. Exclusion criteria were recipients undergoing multiple organ transplantations $(n=6)$ or without RVSP measurement on preoperative 2D-echocardiography $(\mathrm{n}=$ 65). Clinical, laboratory, and outcome data were collected by a trained coordinator using standardized case report protocols from institutional electric medical records. All recipients were analyzed anonymously.

\section{Study endpoints}

The primary endpoint was the correlation between RVSP on preoperative $2 \mathrm{D}$-echocardiography and systolic PAP (sPAP) on intraoperative RHC according to demographic characteristics (sex and body mass index), disease severity (model for end-stage liver disease [MELD] score and presence of ascites), type of LT (living donor or deceased donor), and etiology of disease (cirrhotic or non-cirrhotic). The secondary endpoints were the correlations between RVSP on preoperative 2D-echocardiography and mean pulmonary arterial pressure (mPAP) on intraoperative RHC in the above subgroups.

The positive and negative predictive values of RVSP $>50$ mmHg for identifying mPAP > $35 \mathrm{mmHg}$ and $\mathrm{sPAP}>50$ mmHg were calculated $[4,7]$. Based on intraoperative RHC, recipients with porto-pulmonary hypertension, defined as mPAP $>25 \mathrm{mmHg}$, pulmonary vascular resistance $>3$ Woods units, and pulmonary capillary wedge pressure $<15$ mmHg according to the current guideline [3], were identified, and the baseline characteristics and preoperative treatments in these recipients were reported.

\section{Pulmonary pressure on 2D-echocardiography and RHC}

Following institutional protocol, preoperative 2D-echocardiography was performed in every patient scheduled for LT using various models of commercially available equipment. The following formula was used to calculate RVSP with the assumption of no significant right ventricular outflow tract obstruction:

RVSP $=4 \times(V)^{2}+$ assumed right atrial pressure; 
$V=$ peak velocity of the tricuspid valve regurgitant jet (meters per second);

Right atrial pressure was estimated to be 5,10 , or 15 $\mathrm{mmHg}$ according to diameter and collapsibility of the inferior vena cava [12].

According to institutional protocol, a pulmonary arterial catheter (Edward Lifesciences LLC, USA) was inserted by the attending anesthesiologist and connected to an electronic transducer to measure hemodynamic parameters. To minimize stress, PAP was measured after stabilization from induction of anesthesia but before surgical incision.

\section{Anesthetic care}

Anesthetic care was standardized according to institutional protocol. After applying monitoring devices (peripheral capillary oxygen saturation, five-lead electrocardiography, noninvasive arterial blood pressure), anesthetic induction was achieved with thiopental sodium and maintained with isoflurane. Remifentanil was infused to respond to hemodynamic changes. The respiratory rate was set to achieve normocapnea. Fluids and pressor drugs were infused to maintain mean arterial pressure $70 \mathrm{mmHg}$.

\section{Statistical analysis}

The mean pressures between 2D-echocardiography and RHC were compared using t-test and are presented as mean \pm standard deviation (SD). The correlation was analyzed using Spearman's correlation coefficient and presented as $\mathrm{R}$ and $\mathrm{P}$ values. Scatter plots and Bland-Altman plots for subgroups were generated. Statistical analyses were performed with IBM SPSS Statistics software Version 20.0 (IBM, USA). P values $<0.05$ were considered statistically significant.

\section{RESULTS}

A total of 344 recipients was enrolled for analysis. The median age of recipients was 54 years (interquartile range, 49.2-60.0 years). The median duration between 2D-echocardiography and RHC was 20 days (interquartile range, 13.0-40.8 days). The mean value of RVSP on preoperative 2D-echocardiography was significantly higher than that of sPAP on intraoperative RHC $(27.2 \pm 7.1$ vs. $22.0 \pm 7.3$; P value $<0.001)$. Similar results were observed in most subgroups (Table 1). Correlations of the entire population between RVSP on preoperative 2D-echocardiography and PAP on intraoperative RHC were significant but weak for both sPAP and $\mathrm{mPAP}(\mathrm{R}=0.27 ; \mathrm{P}<0.001$ for $\mathrm{sPAP}, \mathrm{R}=0.24 ; \mathrm{P}<$ 0.001 for mPAP) (Table 2). However, different results were found in some subgroup analyses. In recipients without ascites or with controlled ascites, RVSP on 2D-echocardiography correlated well with both SPAP and MPAP, whereas recipients with uncontrolled ascites showed nonsignificant results $(\mathrm{R}=0.215 ; \mathrm{P}=0.072$ for $\mathrm{sPAP}, \mathrm{R}=0.21 ; \mathrm{P}=0.079$ for $\mathrm{mPAP})$. The correlation also showed inconsistent significance according to type of LT, being significant in recipients of living donor LT but not in those of deceased donor LT ( $\mathrm{R}$ $=0.226 ; \mathrm{P}<0.001$ vs. $\mathrm{R}=0.129 ; \mathrm{P}=0.224$ for $\mathrm{sPAP}, \mathrm{R}=$ $0.193 ; \mathrm{P}=0.002$ vs. $\mathrm{R}=0.163 ; \mathrm{P}=0.126$ for mPAP). Analyses according to disease progression showed that the correlations were significant irrespective of MELD score (Table 2).

A scatter plot of the entire population is shown in Fig. 1. Scatter plots for separate analyses according to type of LT (Fig. 2) and degree of ascites (Fig. 3) are presented. In addition, Bland-Altman plots were generated. According to t-test and regression analysis, the bias between RVSP and sPAP (mean value of RVSP-sPAP) was $5.24 \mathrm{mmHg}$ (SD \pm 8.45 ), and the $95 \%$ limits of agreement were 21.80 and -11.32 mmHg (Fig. 4). For mPAP, the bias between RVSP and mPAP

Table 1. Mean RVSP on 2D-Echocardiography and SPAP on RHC

\begin{tabular}{lccc}
\hline \multicolumn{1}{c}{ Variable } & RVSP & sPAP & P value \\
\hline Overall recipients $(n=344)$ & $27.2 \pm 7.1$ & $22.0 \pm 7.3<0.001$ \\
Male $(n=258)$ & $26.9 \pm 7.2$ & $21.7 \pm 7.4<0.001$ \\
Female $(n=86)$ & $28.2 \pm 6.7$ & $22.9 \pm 6.8<0.001$ \\
BMI $<25(n=247)$ & $27.2 \pm 7.5$ & $21.7 \pm 7.4<0.001$ \\
BMI $\geq 25(n=97)$ & $27.1 \pm 6.122 .7 \pm 6.8<0.001$ \\
MELD $<25(n=247)$ & $27.0 \pm 6.321 .8 \pm 7.1<0.001$ \\
MELD $\geq 25(n=97)$ & $27.7 \pm 9.322 .6 \pm 7.9<0.001$ \\
No ascites $(n=162)$ & $26.1 \pm 5.920 .7 \pm 6.0<0.001$ \\
Ascites $(n=182)$ & $28.1 \pm 8.022 .9 \pm 7.9<0.001$ \\
$\quad$ Controlled ascites $(n=111)$ & $28.8 \pm 8.2$ & $22.8 \pm 8.6<0.001$ \\
$\quad$ Uncontrolled ascites $(n=71)$ & $27.0 \pm 7.5$ & $23.5 \pm 7.2<0.001$ \\
Living donor LT $(n=254)$ & $26.2 \pm 6.5$ & $20.8 \pm 6.2<0.001$ \\
Deceased donor $L T(n=90)$ & $30.1 \pm 7.9$ & $25.3 \pm 8.9<0.001$ \\
Cirrhotic disease $(n=281)$ & $26.3 \pm 6.4$ & $20.6 \pm 5.9<0.001$ \\
HBV related disease $(n=227)$ & $26.6 \pm 6.6$ & $20.7 \pm 6.5<0.001$ \\
HCV related disease $(n=32)$ & $27.4 \pm 7.8$ & $21.4 \pm 6.0<0.001$ \\
Alcoholic disease $(n=50)$ & $27.7 \pm 7.7$ & $24.9 \pm 9.6 \quad 0.110$ \\
Non-cirrhotic disease $(n=63)$ & $31.2 \pm 8.7$ & $28.0 \pm 9.4$ & 0.046 \\
\hline
\end{tabular}

Values are presented as mean \pm SD. RVSP: right ventricular systolic pressure, sPAP: systolic pulmonary arterial pressure, RHC: right heart catheterization, BMI: body mass index, MELD: model for end-stage liver disease, LT: Liver transplantation, HBV: hepatitis $B$ virus, $\mathrm{HCV}$ : hepatitis $C$ virus. 
Table 2. Correlation between RVSP on Echocardiography and PAP on RHC

\begin{tabular}{|c|c|c|c|c|c|}
\hline \multirow{2}{*}{ Variable } & \multirow{2}{*}{ Number (\%) } & \multicolumn{2}{|c|}{ SPAP } & \multicolumn{2}{|c|}{ mPAP } \\
\hline & & $\mathrm{R}$ value & P value & $R$ value & P value \\
\hline Overall recipients & 344 & 0.27 & $<0.001$ & 0.24 & $<0.001$ \\
\hline Male & $258(75)$ & 0.244 & $<0.001$ & 0.184 & 0.003 \\
\hline Female & $86(25)$ & 0.312 & 0.003 & 0.325 & 0.002 \\
\hline $\mathrm{BMI} \leq 25$ & $247(71.8)$ & 0.26 & $<0.001$ & 0.252 & $<0.001$ \\
\hline $\mathrm{BMI}>25$ & $97(28.2)$ & 0.305 & 0.002 & 0.192 & 0.060 \\
\hline MELD $<25$ & $265(76.8)$ & 0.213 & $<0.001$ & 0.18 & 0.003 \\
\hline MELD $\geq 25$ & $79(22.8)$ & 0.446 & $<0.001$ & 0.424 & $<0.001$ \\
\hline No ascites & $162(46.7)$ & 0.213 & 0.006 & 0.155 & 0.049 \\
\hline Ascites & $182(5.3)$ & 0.281 & $<0.001$ & 0.257 & $<0.001$ \\
\hline Controlled ascites & $111(32.3)$ & 0.316 & 0.001 & 0.307 & 0.001 \\
\hline Uncontrolled ascites & $71(20.6)$ & 0.215 & 0.072 & 0.21 & 0.079 \\
\hline Living donor LT & $254(73.8)$ & 0.226 & $<0.001$ & 0.193 & 0.002 \\
\hline Deceased donor LT & $90(26.2)$ & 0.129 & 0.224 & 0.163 & 0.126 \\
\hline Cirrhotic disease & $281(81.7)$ & 0.19 & 0.001 & 0.133 & 0.026 \\
\hline HBV related disease & $227(66)$ & 0.25 & $<0.001$ & 0.17 & 0.010 \\
\hline $\mathrm{HCV}$ related disease & $32(9.3)$ & 0.046 & 0.803 & 0.691 & 0.690 \\
\hline Alcoholic disease & $50(14.5)$ & 0.317 & 0.025 & 0.313 & 0.027 \\
\hline Non-cirrhotic disease & $63(18.3)$ & 0.203 & 0.110 & 0.254 & 0.045 \\
\hline
\end{tabular}

RVSP: right ventricular systolic pressure, PAP: pulmonary arterial pressure, RHC: right heart catheterization, sPAP: systolic PAP, mPAP: mean PAP, BMI: body mass index, MELD: model for end-stage liver disease, LT: Liver transplantation, HBV: hepatitis B virus, HCV: hepatitis C virus.

(mean value of RVSP-mPAP) was $12.79 \mathrm{mmHg}(\mathrm{SD} \pm 7.34$ ), and the $95 \%$ limits of agreement were 27.17 and -1.59 mmHg (Fig. 5).

The positive and negative predictive values of RVSP $>50$ mmHg on echocardiography for identifying mPAP > 35 mmHg were $37.5 \%$ and $49.9 \%$, respectively, and $28.6 \%$ and 49.8\% for sPAP > $50 \mathrm{mmHg}$ (Table 3). According to intraoperative measurements from RHC, five recipients were diagnosed as porto-pulmonary hypertension. In contrast to the results from the other subgroups, preoperative RVSP was lower than SPAP in intraoperative RHC in these recipients $(38.2 \pm 16.5$ vs. $40.6 \pm 8.0)$.

\section{DISCUSSION}

In this study, we evaluated the correlation between RVSP on preoperative 2D-echocardiography and PAP on intraoperative RHC and found a significant but weak correlation in the overall population. In the Bland-Altman plots, the difference between RVSP and PAP tended to be more significant when RVSP or PAP was higher. The subgroup analyses demonstrated that the correlation was not significant in recipients with uncontrolled ascites or in recipients of de-

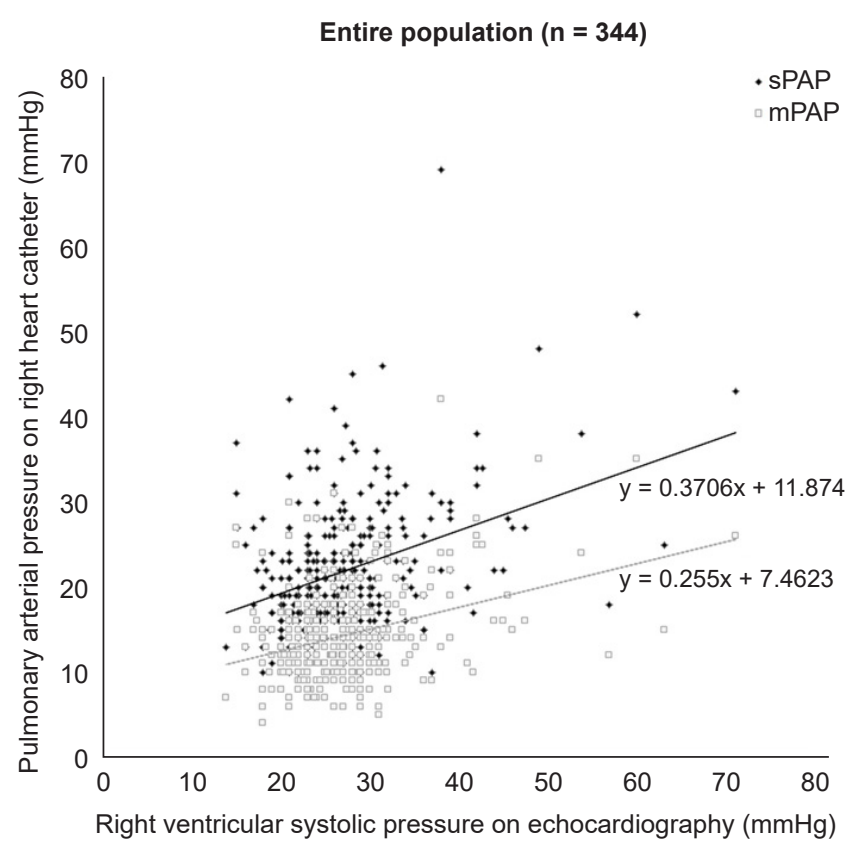

Fig. 1. Scatter plot of the entire population. SPAP: systolic pulmonary arterial pressure, mPAP: mean pulmonary arterial pressure.

ceased donor LT. The results of this study suggest that pulmonary pressure on preoperative $2 \mathrm{D}$-echocardiography 
Living donor liver transplantation $(n=254)$

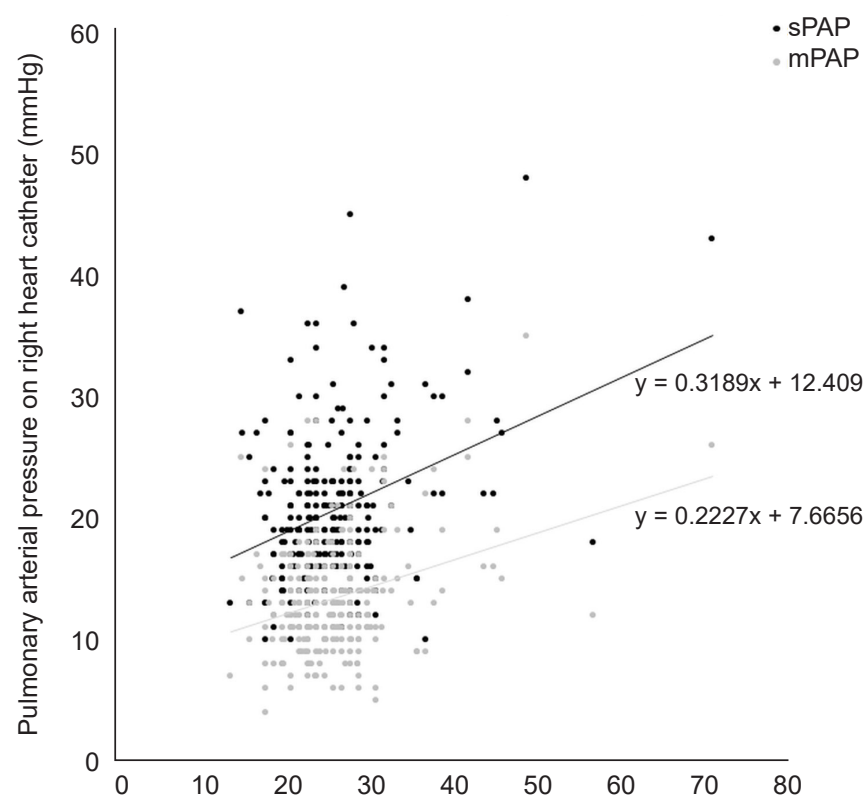

Right ventricular systolic pressure on echocardiography $(\mathrm{mmHg})$
Deceased donor liver transplantation $(n=90)$

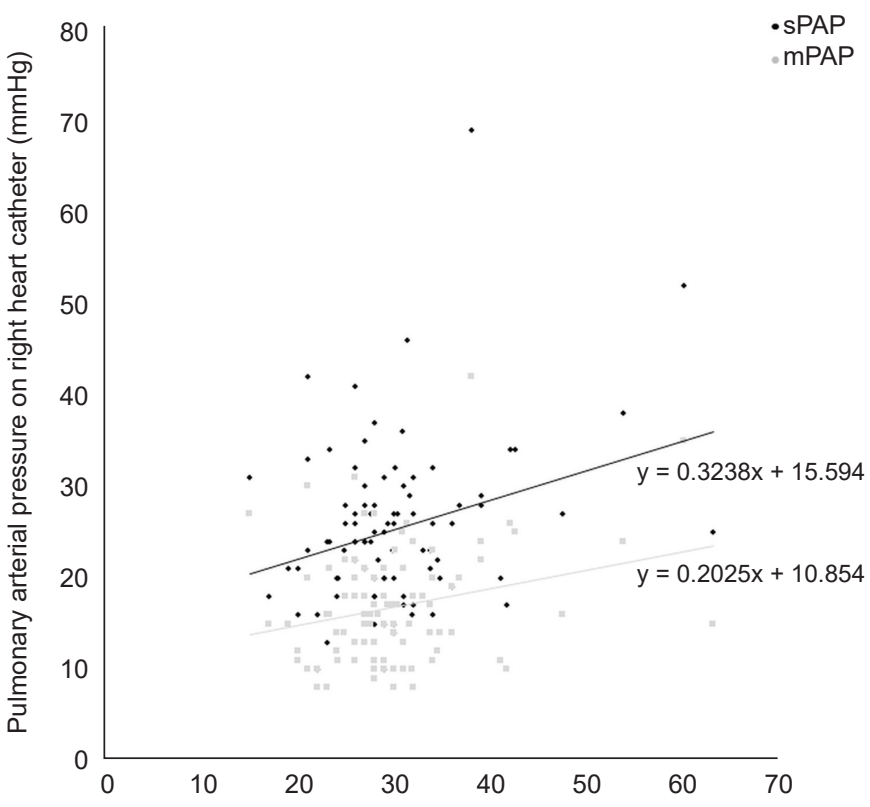

Right ventricular systolic pressure on echocardiography $(\mathrm{mmHg})$

Fig. 2. Scatter plots according to type of liver transplantation. SPAP: systolic pulmonary arterial pressure, mPAP: mean pulmonary arterial pressure.
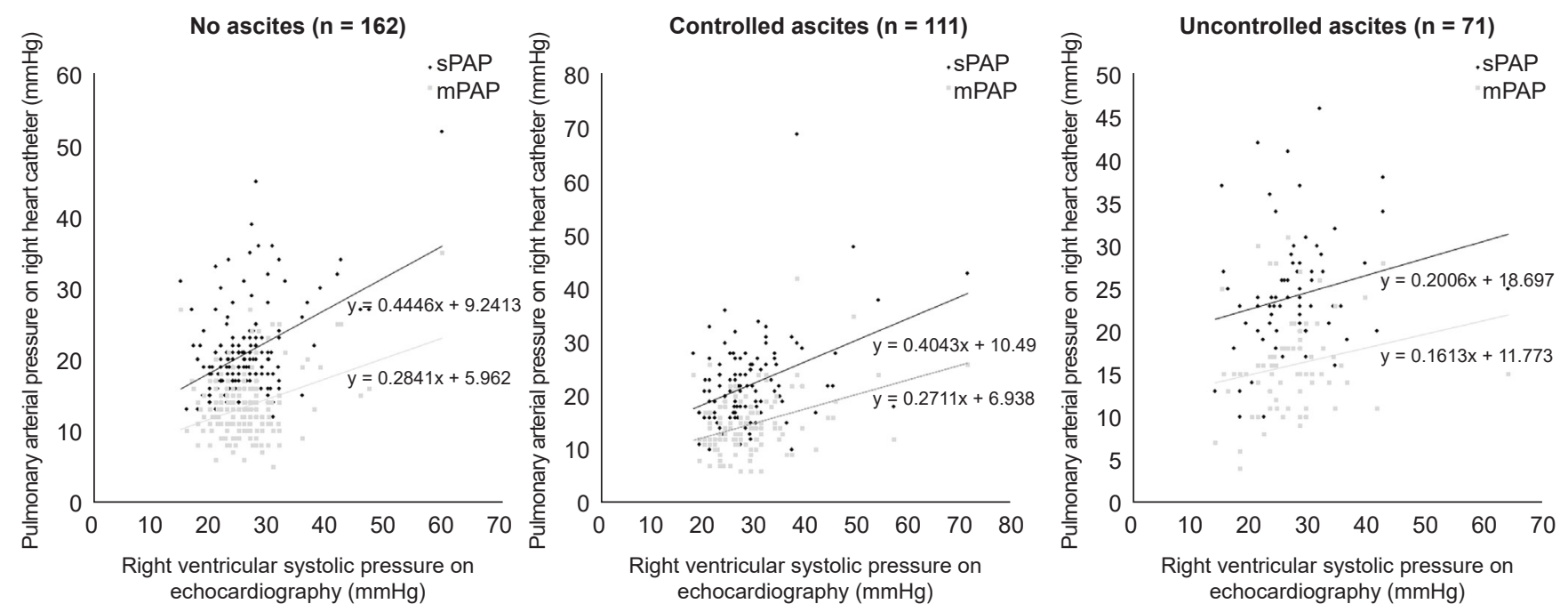

Fig. 3. Scatter plots according to degree of ascites. SPAP: systolic pulmonary arterial pressure, mPAP: mean pulmonary arterial pressure.

does not predict intraoperative state adequately, especially when PAP or RVSP is high or in LT recipients of deceased donor type or with uncontrolled ascites. Moreover, predictive values for identifying $\mathrm{PH}$ with high risk for LT were poor in the entire population and subgroup analysis. Although $2 \mathrm{D}$-echocardiography is an effective modality in screening for porto-pulmonary hypertension in LT candidates, our results question whether preoperative 2D-echocardiography monitoring can reflect intraoperative pulmonary hemodynamics of LT recipients.

Survival after LT is highly dependent on cardiac function, and PAP is directly associated with clinical outcomes of LT $[4,7,13]$. A graded association was shown between mPAP and mortality in the subgroup of patients with high pulmonary vascular resistance, and SPAP was associated with increased risk of hospitalization for cardiac disease [14-16]. 


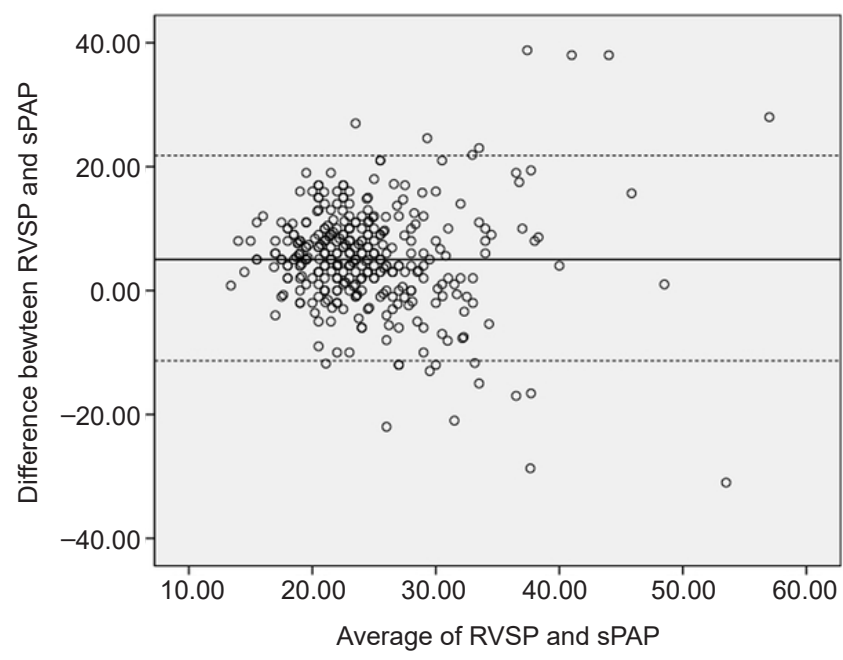

Fig. 4. Bland-Altman plot of systolic right ventricular pressure (RVSP) on preoperative echocardiography to systolic pulmonary arterial pressure (sPAP).

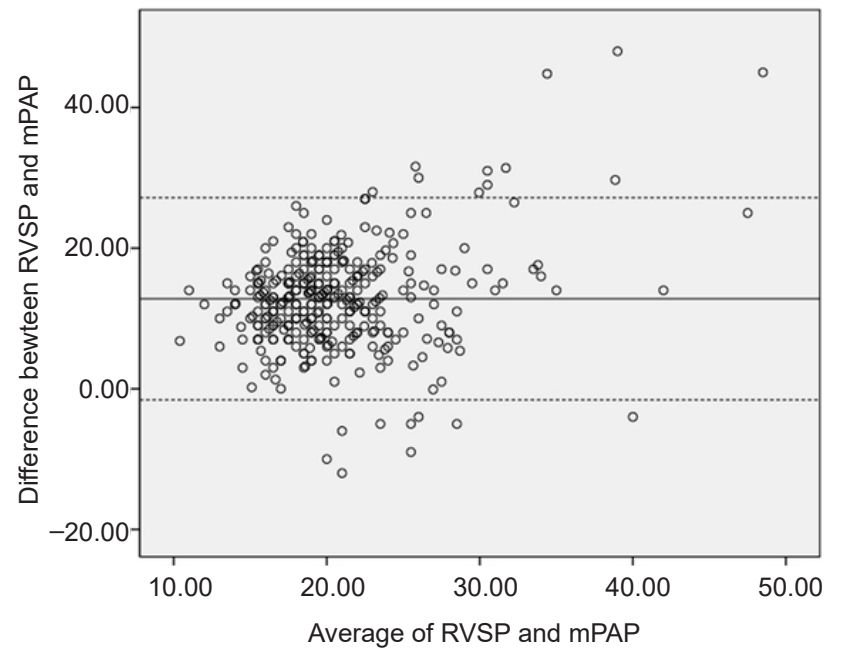

Fig. 5. Bland-Altman plot of systolic right ventricular pressure (RVSP) on preoperative echocardiography to mean pulmonary arterial pressure (mPAP); the bias was $12.79 \mathrm{mmHg}$, and the $95 \%$ limits of agreement were 27.17 and $-1.59 \mathrm{mmHg}$.

Table 3. Predictive Values of RVSP > $50 \mathrm{mmHg}$ to Detect Pulmonary Arterial Hypertension on RHC

\begin{tabular}{|c|c|c|c|c|c|c|c|}
\hline \multirow{2}{*}{ Variable } & \multirow{2}{*}{$\begin{array}{c}\text { RVSP > } 50 \\
m m H g\end{array}$} & \multicolumn{3}{|c|}{ mPAP > $35 \mathrm{mmHg}$ detection } & \multicolumn{3}{|c|}{ sPAP > $50 \mathrm{mmHg}$ detection } \\
\hline & & Number & PPV & NPV & Number & PPV & NPV \\
\hline Overall recipients $(n=344)$ & 5 & 3 & 37.5 & 49.9 & 2 & 28.6 & 49.8 \\
\hline Male $(n=258)$ & 4 & 3 & 42.9 & 49.9 & 2 & 33.3 & 49.8 \\
\hline Female $(n=86)$ & 1 & 0 & 0 & 49.7 & 0 & 0 & 49.7 \\
\hline $\mathrm{BMI}<25(\mathrm{n}=247)$ & 0 & 1 & 100 & 50.1 & 0 & & 50 \\
\hline BMI $\geq 25(n=97)$ & 5 & 2 & 28.6 & 49.2 & 2 & 28.6 & 49.2 \\
\hline MELD < $25(n=247)$ & 4 & 0 & 0 & 49.6 & 0 & 0 & 49.6 \\
\hline MELD $\geq 25(n=97)$ & 1 & 3 & 75 & 50.5 & 2 & 66.7 & 50.3 \\
\hline No ascites $(n=162)$ & 1 & 1 & 50 & 50 & 1 & 50 & 50 \\
\hline Ascites $(n=182)$ & 4 & 2 & 33.3 & 49.7 & 1 & 20 & 49.6 \\
\hline Controlled ascites $(n=111)$ & 3 & 2 & 40 & 49.8 & 1 & 25 & 49.5 \\
\hline Uncontrolled ascites $(n=71)$ & 1 & 0 & 0 & 49.6 & 0 & 0 & 49.6 \\
\hline Living donor LT $(n=254)$ & 2 & 1 & 33.3 & 49.9 & 0 & 0 & 49.8 \\
\hline Deceased donor LT $(n=90)$ & 3 & 2 & 40 & 49.7 & 2 & 40 & 49.7 \\
\hline Cirrhotic disease $(n=281)$ & 3 & 1 & 25 & 49.8 & 0 & 0 & 49.7 \\
\hline HBV related disease $(n=227)$ & 2 & 1 & 33.3 & 49.9 & 0 & 0 & 49.8 \\
\hline HCV related disease $(n=32)$ & 1 & 0 & 0 & 49.2 & 0 & 0 & 49.2 \\
\hline Alcoholic disease $(n=50)$ & 1 & 1 & 50 & 50 & 1 & 50 & 50 \\
\hline Non-cirrhotic disease $(n=63)$ & 2 & 2 & 50 & 50 & 2 & 50 & 50 \\
\hline
\end{tabular}

RVSP: right ventricular systolic pressure, RHC: right heart catheterization, SPAP: systolic pulmonary arterial pressure, mPAP: mean pulmonary arterial pressure, PPV: Positive predictive value, NPV: Negative predictive value, BMI: body mass index, MELD: model for endstage liver disease, LT: Liver transplantation, HBV: hepatitis B virus, HCV: hepatitis C virus.

The use of RHC cannot always be justified as a screening tool due to invasiveness, but it is the only gold standard modality to confirm porto-pulmonary hypertension. Early studies demonstrated that echocardiography can be an effective tool for detecting PH in LT candidates [8,9], and these results have led to wide use of 2D-echocardiography as an initial screening method to determine the need for RHC by screening for cardiac abnormalities or PH. However, predicting intraoperative PAP based on preoperative echocardiographic results can be influenced by other perioperative factors. Therefore, we evaluated the correlation between RVSP on preoperative 2D-echocardiography and PAP on intraopera- 
tive RHC in a large cohort of total LT recipients and its subgroups.

Although several indexes and methods have been adopted to increase accuracy and reproducibility [17], correlations between RVSP on 2D-echocardiography and PAP on RHC have been reported to be weak in the general population [7]. Methods to improve accuracy include measuring tricuspid annular plane systolic excursion, two-dimensional strain, tissue Doppler echocardiography, the speckle tracking method, acceleration time across the pulmonic valve, the pulmonary artery regurgitant jet method, and the tricuspid regurgitant jet method [18]. The most commonly used method is to measure the maximum velocity of the tricuspid regurgitant jet, which was used in this study.

In this study, the correlation between preoperative RVSP on 2D-echocardiography and intraoperative PAP on RHC was significant but weak, and the following inherent limitations might be related to this result. First, 2D-echocardiography was performed in the preoperative period, while PAP was measured intraoperatively by RHC. There is a difference in physiologic status between pre- and intraoperative periods. Second, the echocardiography beam cannot always be parallel to the tricuspid regurgitant jet when obtaining maximum velocity [19]. Third, distal obstruction such as right ventricular outlet obstruction, pulmonic valve stenosis, or supravalvular stenosis might be present [18]. Lastly, the continuous wave Doppler spectrum can be suboptimal or absent. In patients with limited echocardiographic view, contrast agent can be considered to enhance the velocity signal $[20,21]$. Another possibility is related to the time gap from 2D-echocardiography to LT. Due to the retrospective nature of this study, preoperative echocardiographic assessment was not repeated in candidates with normal findings, and this gap was inconsistent among study participants.

Unlike the overall population in which preoperative RVSP far exceeded intraoperative sPAP on RHC, preoperative RVSP slightly underestimated intraoperative sPAP in recipients diagnosed with porto-pulmonary hypertension by RHC. This result corresponds well with previous studies in that, while diagnostic value of $2 \mathrm{D}$-echocardiography is pronounced in patients with moderate to severe PH, a weak correlation with pulmonary pressure was reported among LT candidates overall [7-9]. Our predictive values for identifying clinically significant $\mathrm{PH}$ are lower that those of one previous study [9], owing to the following differences in clinical setting. First, we evaluated the predictive value of preoperative RVSP for identifying intraoperative $\mathrm{PH}$. Unlike a comparison between simultaneous measurements, induction of general anesthesia before catheter insertion as well as the time gap might have affected the results because anesthetics affect pulmonary hemodynamics [22]. Second, the study used a larger cohort and enrolled results of 2D-echocardiography performed in various clinical settings other than echocardiographic laboratories. Echocardiographic imaging is more difficult when related to position of the patient, lack of cooperation, tachypnea or artificial ventilation, and other factors [23]. Inter- and intra-observer variability of echocardiographic assessment can be pronounced in less experienced hands [24]. The strength of this study is that the results reflect the correlations of real-world practice in various clinical settings of LT recipients and are presented according to these subgroups.

In recipients with ascites, only the group with uncontrolled ascites showed a non-significant correlation. This result could be related to increased intra-abdominal pressure that might have affected right atrial pressure by altering venous return [25]. Previous studies on the effect of increased intra-abdominal pressure on the circulatory system have reported inconsistent results. While initial studies assumed that venous return would increase following intra-abdominal pressure, subsequent studies showed a decreased venous return [26]. To explain these contradictory results, a hypothesis that vascular waterfall occurs in the inferior vena cava at diaphragm level was proposed. The waterfall phenomenon was demonstrated in an animal study and is presumed to interact with intra-abdominal pressure, inferior vena cava pressure, and transmural closing pressure of the inferior vena cava [27].

Although 2D-echocardiography can be clinically valuable in screening PH in LT candidates, our findings suggest that preoperative $2 \mathrm{D}$-echocardiography might not be sufficient for predicting intraoperative state. That is because some of the clinical settings in this study are inevitable; moreover, diagnostic criteria of porto-pulmonary hypertension require direct measurements from RHC such as mPAP, pulmonary vascular resistance, and pulmonary capillary wedge pressure. Thus, it is reasonable to consider intraoperative RHC actively for recipients at risk. Also, selection of LT candidates for preoperative RHC and measures to improve quality of preoperative $2 \mathrm{D}$-echocardiography is an important issue that was not resolved by this study. The efficacy of intraoperative echocardiography and the correlation with RHC during LT procedures are beyond the scope of this study and require further randomized investigations. 
Our study was limited by its retrospective design and potential selection bias. Different time intervals from preoperative 2D-echocardiography to surgery also might have caused variability. Sensitivity and specificity for detecting PH could not be analyzed because patients with marked elevation of PAP were allocated for transplantation only after successful treatment. Lastly, the association with clinical outcome was not analyzed in this study. Despite these limitations, this is the first study validating preoperative 2D-echocardiography for predicting intraoperative PAP in LT recipient subgroups.

In LT recipients, the correlations between RVSP on preoperative 2D-echocardiography and PAP on intraoperative RHC are significant but weak. Preoperative 2D-echocardiography might not be reliable in predicting intraoperative pulmonary hemodynamics, and it is reasonable to consider intraoperative $\mathrm{RHC}$ for recipients at risk of $\mathrm{PH}$.

\section{CONFLICTS OF INTEREST}

No potential conflict of interest relevant to this article was reported.

\section{DATA AVAILABILITY STATEMENT}

The datasets generated during and/or analyzed during the current study are available from the corresponding author on reasonable request.

\section{AUTHOR CONTRIBUTIONS}

Conceptualization: Jungchan Park, Myung Soo Park, Gaab Soo Kim. Formal analysis: Keoungah Kim. Funding acquisition: Gaab Soo Kim. Methodology: Seung-Hwa Lee, Gaab Soo Kim. Project administration: Ah Ran Oh, Gyu-Seong Choi. Visualization: Myung Soo Park, Ji-Hye Kwon, Keoungah Kim. Writing - original draft: Jungchan Park, Myung Soo Park. Writing - review \& editing: Gaab Soo Kim. Investigation: Myung Soo Park, Gyu-Seong Choi. Resources: Jungchan Park. Software: Ah Ran Oh. Supervision: Seung-Hwa Lee, Jong Man Kim, Gaab Soo Kim. Validation: Jong Man Kim.

\section{ORCID}

Jungchan Park, https://orcid.org/0000-0002-7794-3547

Myung Soo Park, https://orcid.org/0000-0001-7832-4513

Ji-Hye Kwon, https://orcid.org/0000-0002-8899-0699
Ah Ran Oh, https://orcid.org/0000-0002-8076-5104

Seung-Hwa Lee, https://orcid.org/0000-0001-5508-7519

Gyu-Seong Choi, https://orcid.org/0000-0003-2545-3105

Jong Man Kim, https://orcid.org/0000-0002-1903-8354

Keoungah Kim, https://orcid.org/0000-0002-4924-1046

Gaab Soo Kim, https://orcid.org/0000-0002-9383-2652

\section{REFERENCES}

1. Salgia RJ, Goodrich NP, Simpson H, Merion RM, Sharma P. Outcomes of liver transplantation for porto-pulmonary hypertension in model for end-stage liver disease era. Dig Dis Sci 2014; 59: 1976-82

2. Houlihan DD, Holt A, Elliot C, Ferguson JW. Review article: liver transplantation for the pulmonary disorders of portal hypertension. Aliment Pharmacol Ther 2013; 37: 183-94.

3. Krowka MJ, Fallon MB, Kawut SM, Fuhrmann V, Heimbach JK, Ramsay MA, et al. International Liver Transplant Society Practice Guidelines: diagnosis and management of Hepatopulmonary syndrome and Portopulmonary hypertension. Transplantation 2016; 100: 1440-52.

4. Krowka MJ, Plevak DJ, Findlay JY, Rosen CB, Wiesner RH, Krom RA. Pulmonary hemodynamics and perioperative cardiopulmonary-related mortality in patients with portopulmonary hypertension undergoing liver transplantation. Liver Transpl 2000; 6: 443-50.

5. Gwak MS, Kim JA, Kim GS, Choi SJ, Ahn H, Lee JJ, et al. Incidence of severe ventricular arrhythmias during pulmonary artery catheterization in liver allograft recipients. Liver Transpl 2007; 13: 1451-4.

6. Galiè N, Humbert M, Vachiery JL, Gibbs S, Lang I, Torbicki A, et al. 2015 ESC/ERS Guidelines for the diagnosis and treatment of pulmonary hypertension: The Joint Task Force for the Diagnosis and Treatment of Pulmonary Hypertension of the European Society of Cardiology (ESC) and the European Respiratory Society (ERS): endorsed by: Association for European Paediatric and Congenital Cardiology (AEPC), International Society for Heart and Lung Transplantation (ISHLT). Eur Respir J 2015; 46: 903-75

7. Janda S, Shahidi N, Gin K, Swiston J. Diagnostic accuracy of echocardiography for pulmonary hypertension: a systematic review and meta-analysis. Heart 2011; 97: 612-22.

8. Kim WR, Krowka MJ, Plevak DJ, Lee J, Rettke SR, Frantz RP, et al. Accuracy of Doppler echocardiography in the assessment of pulmonary hypertension in liver transplant candidates. Liver Transpl 2000; 6: 453-8.

9. Cotton CL, Gandhi S, Vaitkus PT, Massad MG, Benedetti E, 
Mrtek RG, et al. Role of echocardiography in detecting portopulmonary hypertension in liver transplant candidates. Liver Transpl 2002; 8: 1051-4.

10. Sussman N, Kaza V, Barshes N, Stribling R, Goss J, O’Mahony C, et al. Successful liver transplantation following medical management of portopulmonary hypertension: a single-center series. Am J Transplant 2006; 6: 2177-82.

11. Ashfaq M, Chinnakotla S, Rogers L, Ausloos K, Saadeh S, Klintmalm GB, et al. The impact of treatment of portopulmonary hypertension on survival following liver transplantation. Am J Transplant 2007; 7: 1258-64.

12. Rudski LG, Lai WW, Afilalo J, Hua L, Handschumacher MD, Chandrasekaran K, et al. Guidelines for the echocardiographic assessment of the right heart in adults: a report from the American Society of Echocardiography endorsed by the European Association of Echocardiography, a registered branch of the European Society of Cardiology, and the Canadian Society of Echocardiography. J Am Soc Echocardiogr 2010; 23: 685-713; quiz 786-8.

13. Krowka MJ, Mandell MS, Ramsay MA, Kawut SM, Fallon MB, Manzarbeitia C, et al. Hepatopulmonary syndrome and portopulmonary hypertension: a report of the multicenter liver transplant database. Liver Transpl 2004; 10: 174-82.

14. Kia L, Shah SJ, Wang E, Sharma D, Selvaraj S, Medina C, et al. Role of pretransplant echocardiographic evaluation in predicting outcomes following liver transplantation. Am J Transplant 2013; 13: 2395-401.

15. Bushyhead D, Kirkpatrick JN, Goldberg D. Pretransplant echocardiographic parameters as markers of posttransplant outcomes in liver transplant recipients. Liver Transpl 2016; 22: 316-23.

16. Raevens S, Colle I, Reyntjens K, Geerts A, Berrevoet F, Rogiers X, et al. Echocardiography for the detection of portopulmonary hypertension in liver transplant candidates: an analysis of cutoff values. Liver Transpl 2013; 19: 602-10.

17. Milan A, Magnino C, Veglio F. Echocardiographic indexes for the non-invasive evaluation of pulmonary hemodynamics. J Am Soc Echocardiogr 2010; 23: 225-39; quiz 332-4.

18. Hemnes AR, Forfia PR, Champion HC. Assessment of pulmonary vasculature and right heart by invasive haemodynamics and echocardiography. Int J Clin Pract Suppl 2009; (162):4-19.

19. Posteraro A, Salustri A, Trambaiolo P, Amici E, Gambelli G. Echocardiographic estimation of pulmonary pressures. J Cardiovasc Med (Hagerstown) 2006; 7: 545-54.

20. Jeon DS, Luo H, Iwami T, Miyamoto T, Brasch AV, Mirocha J, et al. The usefulness of a $10 \%$ air- $10 \%$ blood- $80 \%$ saline mixture for contrast echocardiography: Doppler measurement of pulmonary artery systolic pressure. J Am Coll Cardiol 2002; 39: 124-9.

21. Waggoner AD, Barzilai B, Pérez JE. Saline contrast enhancement of tricuspid regurgitant jets detected by Doppler color flow imaging. Am J Cardiol 1990; 65: 1368-71.

22. Fischer LG, Aken HV, Bu Combining Diaeresis Rkle H. Management of pulmonary hypertension: physiological and pharmacological considerations for anesthesiologists. Anesth Analg 2003; 96: 1603-16.

23. Goodkin GM, Spevack DM, Tunick PA, Kronzon I. How useful is hand-carried bedside echocardiography in critically ill patients? J Am Coll Cardiol 2001; 37: 2019-22.

24. Ling LF, Obuchowski NA, Rodriguez L, Popovic Z, Kwon D, Marwick TH. Accuracy and interobserver concordance of echocardiographic assessment of right ventricular size and systolic function: a quality control exercise. J Am Soc Echocardiogr 2012; 25: 709-13.

25. Dagar G, Taneja A, Nanchal RS. Abdominal circulatory interactions. Crit Care Clin 2016; 32: 265-77.

26. Wexler L, Bergel DH, Gabe IT, Makin GS, Mills CJ. Velocity of blood flow in normal human venae cavae. Circ Res 1968; 23: 349-59.

27. Takata M, Wise RA, Robotham JL. Effects of abdominal pressure on venous return: abdominal vascular zone conditions. J Appl Physiol (1985) 1990; 69: 1961-72. 